\title{
UNA MIRADA DESDE LO LOCAL: APROXIMACIÓN A LA ESTRUCTURA DE LA INFORMACIÓN EN HUELVA (2006-2008)
}

\author{
$\mathrm{M}^{\mathrm{a}}$ Ángeles Pastor Alonso \\ m.a.pastor@ono.com
}

\begin{abstract}
Resumen: Se muestra en este trabajo una panorámica de los medios de comunicación en la ciudad de Huelva en un momento especialmente significativo de su historia reciente, sin obviar los antecedentes que ayudan a entender los cambios producidos. Están presentes los medios vinculados a grandes empresas de comunicación, y también pequeñas estructuras informativas en prensa y radio que aportan novedades representativas. En un punto medio de formalización se encuentran las televisiones locales, inmersas en un proceso de reconversión tecnológica que saca a la luz otros conflictos e intereses. Desde este enfoque local pueden identificarse tendencias que operan en los procesos de comunicación más complejos.
\end{abstract}

Palabras clave: Estructura, local, información, Huelva

Abstract: This paper shows a panoramic view of the mass media in Huelva in a specially significant moment of its recent history, without missing the antecedents which help us to understand the changes have occurred. Not only the media linked with big communication companies are present in this paper, but also some small informative press and radio structures which introduce new representative aspects. Half way between them we find the local television stations, inmersed into a technological adaptation process in which new conflicts and interests arise. From this local point of view, it is possible to identify tendencies to the ones which occur in more complex communication processes.

Keywords: Structure, local, information, Huelva

\section{INTRODUCCIÓN}

a prensa onubense, la más modesta de las andaluzas, es también la menos conocida o estudiada". Así lo constataba el clásico estudio de Checa Godoy (1991: 709) sobre el recorrido histórico de la prensa en Andalucía, y desde entonces el panorama no ha variado mucho. En cualquiera de los aspectos referidos al campo de la comunicación en Huelva -historia de los medios, géneros, empresas informativas, análisis del discurso, etc.-, escasas o nulas son las referencias que pueden encontrarse.

Este trabajo quisiera contribuir modestamente a paliar alguna de esas carencias. Tiene un objetivo tan simple como complejo: esbozar un mapa aproximado de la 

información en Huelva (2006-2008)

estructura de la información en la ciudad de Huelva, una pequeña capital de provincia de casi 150.000 habitantes, e identificar en este nivel geográficamente reducido las mismas tendencias que operan en los procesos comunicativos a nivel mundial.

La realidad de los medios de comunicación en Huelva tiene, por supuesto, características propias relacionadas con la estructura social y económica de la ciudad, con los destinatarios de los contenidos de esos medios y con la propia historia local. La extensión otorgada en este artículo a cada uno de ellos es proporcional a su relevancia y ejemplaridad para mostrar algunas de las tendencias de la comunicación a que nos referimos. Recogemos también otras iniciativas comunicativas independientes que consiguen, en el entorno de lo local, gozar de cierta repercusión. Es precisamente en el ámbito de estas microestructuras donde se aprecian más novedades y perspectivas estimulantes, y donde hay que buscar las claves que ayuden a plantear cómo podría ser la información del futuro.

\section{PRENSA}

La historia de la prensa en Huelva es prolija en cabeceras ya desaparecidas. Fue Odiel, perteneciente a la prensa del movimiento, el único periódico provincial durante cuatro décadas, pero pese al crecimiento urbano e industrial de la capital durante ese periodo, no fue nunca un diario de amplia lectura: apenas superaba los 5.000 ejemplares en los años setenta. Este dato indica ya una característica del panorama de la prensa escrita onubense: la escasez de lectores ha sido siempre un baldón difícil de superar.

Odiel cerró sus puertas - para el recuerdo queda la vieja redacción de la calle Marina, donde convivían redactores y linotipistas en el mismo espacio- en 1984, cuando ya estaba en la calle el periódico llamado a sucederle, Huelva Información. Tras la extinción del Odiel hubo en la provincia una efímera aventura periodística a cargo del diario La Noticia, dirigido por José Ma Allas, que no llegó a diez meses de vida. "La empresa promotora, de escasos recursos y talante progresista cercano al PSOE, no pudo resistir las inevitables pérdidas de los primeros tiempos" y cerró en mayo de 1985, aunque sectores ideológicamente próximos anunciaban poco después su intención de relanzar el viejo Odiel (Checa Godoy, 1991: 450).

Otro intento de cabecera local no consolidado fue el diario La voz de Huelva (1995-2000), una iniciativa promovida por el empresario Nicolás González tras un enfrentamiento con los socios de Huelva Información que se salda con la edición de su propia cabecera. La Voz de Huelva estaba integrada en la cadena de Diario 16 mediante un contrato de franquicia (Egea, 2001). Pese a los intentos por mantenerse a flote, no pudo hacerse un hueco en el panorama informativo de la provincia $^{33}$. En sus últimos años la competencia fue feroz: se lanzaron dos nuevos

\footnotetext{
${ }^{33}$ Como muestra del buen hacer periodístico del diario La voz de Huelva queda su colección fotográfica, de enorme interés para la investigación de la historia local, que ha sido comprada recientemente, a precio simbólico, por la Diputación de Huelva para incorporarla a su archivo.
} 
rotativos, Odiel Información, que no tardaría en vincularse a una gran cadena de comunicación, y La prensa de Huelva, promovida por empresarios onubenses ${ }^{34}$.

Mientras tanto, el diario de referencia provincial, Huelva Información, se vinculaba con diversos grupos editoriales, en un apresurado cambio de manos. Durante un corto espacio de tiempo hubo en Huelva cuatro diarios locales, una eclosión que el mercado era incapaz de absorber, y al igual que La voz de Huelva, también desapareció La Prensa. Poco después el empresariado onubense puso en marcha la edición local de El Mundo junto a Unedisa. Así que los diarios que hoy día se mantienen, aún con pérdidas, están ligados de una manera u otra a fuertes empresas en el mundo de la comunicación, una tendencia generalizada a nivel mundial.

Huelva Información, el decano de la prensa onubense, es también el diario de más difusión de la provincia ${ }^{35}$, pese a los cambios en su accionariado durante este primer cuarto de siglo de existencia -su primer número salió a la calle el 3 de agosto de 1983, fiesta local en Huelva-. Fruto de la iniciativa de un grupo de empresarios, su Consejo de Administración estuvo primero dominado por la Federación Onubense de Empresarios ( $F O E)$, en estrecha alianza, implícita y explícita, con la Asociación de Industrias Químicas y Básicas (AIQB), de la que dependen muchos de los parámetros socioeconómicos de Huelva y su provincia. Era normal que estos sectores pretendieran contar con un aliado informativo en una época en que el desarrollo económico se afianzó pero en la que también surgieron numerosos conflictos medioambientales, lo que repercutió sin duda en el proceso de selección y tratamiento de las noticias. Juan Manuel Tellechea ha estudiado cómo

los responsables del periódico trataban de conjugar los intereses del accionariado, el poder fáctico que representaban las industrias químicas y el deterioro medioambiental por el efecto contaminador, con la información veraz a la que todo ciudadano tiene derecho, según recoge la Constitución española. A ello se unía la presión ejercida por el notable nivel de publicidad que las industrias químicas volcaban en Huelva Información, lo cual condicionaba las actitudes informativas (Tellechea, 2004: 319).

Con estos presupuestos Tellechea llega a la conclusión de que, en esa primera década de existencia del periódico, "la libertad expresiva estaba delimitada, autocensurada y subordinada a presiones internas y externas". Una historia que ha vuelto a repetirse, quizás no de forma tan aguda, con el actual debate público en torno a la industria y el desmantelamiento del Polo Químico. Puesto que su más directo competidor, El Mundo-Huelva Noticias, se encuentra editorialmente

\footnotetext{
${ }^{34} \mathrm{La}$ Prensa de Huelva, publicación de marcado sesgo empresarial que atendía de forma preferente la información económica local y provincial, fue editada por Soporte y Medios de Comunicación de Huelva, constituida al $100 \%$ de capital privado. Esta editora tenía en sus manos también la publicación del semanario de información general 7 Días de Huelva.

${ }^{35}$ Según los datos de la OJD (www.introl.es), el promedio de tirada de julio de 2006 a junio de 2007 es de 8.440 ejemplares: casi iguala la de los dos diarios de la competencia juntos.
} 

información en Huelva (2006-2008)

posicionado junto a la AIQB, desde Huelva Información se dio al principio máxima cabida a la información que generaba la plataforma ciudadana Mesa de la Ría, y hubo cierto apoyo tácito a sus planteamientos. Una línea informativa que no se ha visto continuada, pues la plataforma ha denunciado en repetidas ocasiones el silenciamiento de sus propuestas en los medios de comunicación onubenses. Para explicar este viraje parece sensato no descartar las presiones publicitarias que haya recibido el periódico.

Tras una crisis en el seno del accionariado fundador, Huelva Información pasó, desde 1996, por las manos de varios grupos de comunicación: Prensa Española, Grupo Correo (hoy Vocento), y desde 2002, el Grupo Joly, como parte de su política de expansión en la prensa regional andaluza que cuenta en la actualidad con ocho cabeceras locales. Hacia el año 2000 Joly tenía proyectos de poner en marcha una cabecera en la provincia para consolidar así su presencia en Andalucía occidental, liderada por el Diario de Cádiz. Entonces, recordémoslo, el panorama de la prensa en Huelva estaba totalmente saturado. En lugar de iniciar un nuevo diario, el grupo Joly supo esperar el momento propicio y se hizo con el control de Huelva Información. Ello significó que el periódico renunciara a la mayor parte de sus colaboradores locales, que fueron sustituidos por otros vinculados al grupo: el resultado es una línea editorial más homogénea, pero también más cerrada a los propios intereses de los ciudadanos de Huelva. Como en los demás periódicos de Joly, hay una edición digital (www.huelvainformación.es) que sitúa al diario por delante de sus competidores (la de Odiel es muy reciente y Huelva Noticias aún carece de ella).

Desde que Joly asumió la titularidad ha habido dos directores: Ramón Ramos, actualmente en Granada Hoy, y Antonio Castro Caro, que perteneció a la plantilla de los "fundadores" y, tras un periplo profesional por otros medios, se reincorporó a Huelva Información en 2003. Lógicamente también ha habido cambios en la ubicación del periódico: ocupó primero unos locales en la calle Marina, cerca de donde había tenido su sede el Odiel; luego pasó a la Plaza de San Pedro, y en 2004, ya formando parte del grupo Joly, inaugura una remozada redacción en la Avenida Montenegro, precisamente al lado del complejo de industrias que durante estas décadas ha proyectado su sombra sobre el tratamiento informativo del diario.

Cumplidos ya sus primeros veinticinco años, Huelva Información mantiene un cómodo liderazgo sobre sus rivales a pesar del preocupante descenso de lectores de los últimos tiempos. Como los hábitos de lectura en prensa local son tan difíciles de cambiar, haría falta un lapso más amplio para que las tendencias al alza de otros medios se consoliden. Por cierto, en este diario se han formado ya varias generaciones de profesionales de la información que luego han nutrido la demanda de cuadros en el resto de periódicos locales.

Uno de ellos, el siguiente por orden cronológico, es Odiel Información, que sale a la luz el 14 de febrero de 1999: aquel proyecto de recuperar la histórica cabecera del Odiel del que hablaban los editores del efímero periódico La noticia 
de Huelva, tardó más de diez años en hacerse realidad. La empresa editora era Odiel Press SL, que continúa siendo titular del medio aunque haya cambiado varias veces de dueño.

La deriva de la titularidad del periódico está asociada a la suerte que corrieron otros medios andaluces agrupados en Prensa Sur, compañía a la que se relaciona con el PSOE de Andalucía: primero fue adquirida prácticamente en su totalidad por el Nuevo Grupo de Comunicación Andaluza, del empresario Emilio Martín, editor y principal accionista de El Correo de Andalucía, que intenta sentar las bases para la constitución de un grupo multimedia en la comunidad del sur de España. Y más tarde pasa a la órbita del Grupo Prisa, que en esa época estaba realizando una apuesta por los medios regionales y locales. En octubre de 1999 Polanco obtiene el 75\% de Espacio Editorial, que integra Prensa Sur junto a otras empresas: entre ellas, Odiel Press (Egea, 2001). El diario fue el primero de la provincia en sufrir lo que viene siendo una constante en el mercado de la prensa local: la integración en grandes grupos mediáticos ${ }^{36}$.

El primer director del Odiel fue J.L. Jerez Manfredi, un periodista de dilatada trayectoria que actualmente continúa vinculado a otros medios de la capital. Tras un periodo de transición al frente de Miguel Ortega, en que se incluye por fin un editorial sobre temas locales, el diario queda absorbido por el potente monopolio de los contenidos de El Correo; de hecho, su director pasa a ser el mismo que el del diario sevillano, Fernando Orgambide, quedando en Huelva sólo un delegado del medio, igual que en el resto de periódicos de Prisa en Andalucía. Esta decisión empresarial inicia una estrategia de riguroso ajuste de costes, con la consecuente reducción de plantilla. El Correo de Andalucía se convirtió en el centro editorial desde el que se procesaba, elaboraba y distribuía la información de ámbito internacional, nacional y regional para el resto de los diarios regionales del Grupo, un sistema que, según reconocía la empresa en su memoria económica de 2002, permitía "mejorar la calidad del producto al aglutinar esfuerzos y dedicar los recursos propios de cada diario al ámbito informativo diferenciador, que es el local"37.

Sin embargo, esta política de centralizar lo más posible la gestión no resultó muy acertada. El número de páginas locales disminuyó, los columnistas de Huelva prácticamente desaparecieron, las promociones eran las mismas que en los demás periódicos andaluces -se trató de incrementar el número de ventas casi a golpe de obsequios que funcionaran como atractivo comercial para la clientela- y se dependía de Sevilla para todo. La renuncia a una percepción de la información más propia y cercana pasó factura: el número de lectores descen-

\footnotetext{
${ }^{36}$ Además de Odiel Información, Prisa llegó a controlar hasta cinco periódicos en el panorama andaluz a través de su filial GMI (Grupo Empresarial de Medios Impresos): El Correo de Andalucía, La voz de Almería, el Diario de Jaén y un efímero El Correo de Málaga.

${ }^{37} \mathrm{Cf}$. http://www.prisa.es/static/es/accionistas/memoria2002/unidades_prensa.html (memoria del ejercicio de 2002).
} 

información en Huelva (2006-2008)

dió drásticamente, aunque se pretendieran mantener los ingresos publicitarios a costa de incrementar las suscripciones gratuitas. Sin duda este fracaso tiene que ver, también, con hábitos poderosamente asentados en la prensa andaluza, que no cuenta con un modelo firme de cabecera regional, pero sí con "una fuerte implantación de la prensa de carácter local que supera también en mucho a las cifras de venta de los periódicos de ámbito estatal" (Reig, 1998: 104). Fue la particular travesía del desierto del diario onubense, que sólo logró sobrevivir por el respaldo de un grupo empresarial fuerte.

En Septiembre de 2005 GMI procede a relanzar el periódico, que iba acumulando alarmantemente pérdidas, corrigiendo estrategias equivocadas. Estrena nueva maqueta, con mancheta inspirada en la del anterior Odiel; se proyectan nuevos contenidos y, sobre todo, hay movimientos en la cúpula directiva: Luis Eduardo Siles, un hombre muy vinculado al grupo Prisa que hasta entonces había ejercido de delegado del medio, pasa a ser director; se trae como subdirector a Antonio Peinazo, que era redactor jefe de Huelva Información, y Javier García Salas, otro periodista onubense, es nombrado redactor jefe. A partir de esta apuesta por adaptar el diario a sus receptores y priorizar contenidos locales la progresión fue constante. Aunque se mantuvieron páginas comunes de información nacional e internacional con El Correo, y la rotativa continuó en Sevilla, la mayor autonomía en la gestión fue produciendo los frutos esperados. Las pérdidas acumuladas se cifran en torno a los tres millones de euros y la distancia con Huelva Información todavía es enorme, pero durante ese periodo se aprecia un incremento del número de lectores al tiempo que desciende levemente la difusión del periódico de Joly. Se marca, pues, una tendencia que revaloriza el medio frente a sus competidores ${ }^{38}$.

Pero la coyuntura empresarial de Prisa se estaba gestando en sentido contrario. En otoño de 2006, quizás por la necesidad de impulsar otras áreas de actividad -por ejemplo, su nuevo canal de televisión-, el grupo decide que la prensa regional no es un objetivo estratégico en ese momento. Ignacio de Polanco, recién nombrado sucesor, toma una de sus primeras decisiones importantes al afrontar las desinversiones de su negocio de prensa regional. El primer movimiento en este sentido fue la venta de Odiel Press, en diciembre de 2006, a la que siguieron el resto de diarios andaluces durante 2007. En el caso de Odiel los compradores fueron el Grupo Industrial Gallardo junto con tres empresarios de Huelva, cada uno con una participación del $25 \%$. Los nuevos accionistas ratificaron a toda la estructura directiva y al resto de los trabajadores -alrededor de 40- en sus puestos, puesto que consideraban que eran los que habían sacado el producto a flote. El hecho de conservar a sus responsables es coherente con el mantenimiento

\footnotetext{
${ }^{38}$ Según la OJD, en el periodo de 2005 a 2006 el promedio de tirada de Odiel fue de 5.001 ejemplares diarios, la más alta de su historia; el año siguiente (julio 2006-junio 2007) la cifra es algo más baja, 4.586 ejemplares. Curiosamente, y al contrario que los otros dos diarios locales, la difusión de los domingos es menor que la del resto de días de la semana: ello se explica por la cantidad de suscripciones gratuitas que incrementan la tirada. Cf. www.introl.es.
} 
de la línea editorial: Prisa sigue perteneciendo al Consejo de Administración de Odiel Press de forma simbólica, y el diario mantiene la vinculación y frecuentes colaboraciones con otras empresas del Grupo.

Pero, ¿cuáles son los intereses de estos nuevos dueños en el periódico onubense? A Alfonso Gallardo, primer industrial extremeño y decimotercero de toda España, se le ha vinculado repetidamente con la Junta de Extremadura, de la que ha recibido numerosas subvenciones, pero su relación con el grupo Prisa tampoco es nueva ${ }^{39}$. En cuanto a los empresarios onubenses, el más conocido es Francisco Urbano, presidente de UPECO (Unión Provincial de Empresarios de la Construcción), que ha pasado de ser un desconocido a formar parte de la galería habitual de fotografiados en Odiel y a tomar posiciones en el entramado socioeconómico onubense. La UPECO, que al menos tiene la excusa de ser una institución provincial, ha encontrado en este diario su más eficaz gabinete de prensa.

Por su parte, el grupo empresarial extremeño -centrado en el sector siderúrgico- anunció que la compra no estaba relacionada con una ampliación de actividades, sino que la entrada en prensa sería "puntual" porque el Grupo Gallardo tiene intereses en la provincia. Los hechos han desmentido tajantemente esas declaraciones: meses después el Grupo Gallardo se hizo con el $100 \%$ de las acciones de El Correo de Andalucía y con la mayoría de las de Diario de Jaén, y en junio de 2008 firma un preacuerdo para la adquisición del $80 \%$ del Grupo Zeta $^{40}$. Desde la perspectiva de lo local que aquí se plantea, las suspicacias que han levantado estas operaciones están relacionadas, naturalmente, con el proyecto del industrial de construir una refinería en Tierra de Barros, cuyas centrales de recepción del crudo se instalarían en el puerto onubense; por la provincia de Huelva transcurriría, además, la mayor parte del trazado del oleoducto que trasladaría el crudo hasta la planta extremeña. Por supuesto, el oleoducto ha sido atención preferente de las páginas de Odiel, que durante el último año no ha cesado de proclamar sus bondades ${ }^{41}$. El debate público en torno a este asunto ha sido permanentemente alentado por los otros dos periódicos locales: la edición local de El Mundo, ideológicamente posicionada frente a Odiel, y Huelva Información, que tampoco está libre de sospecha por las inversiones publicitarias que recibe de CEPSA, empresa directamente perjudicada por el proyecto de Gallardo. Vienen a cuento las palabras de E. Bustamante citadas por Reig (1998: 157): "la

\footnotetext{
${ }^{39} \mathrm{Ambas}$ empresas eran los accionistas mayoritarios de Productora Extremeña de Información S.A. (Petsa), que fue la adjudicataria inicial del proyecto de televisión autonómica de Extremadura, en 2001. Posteriormente la Junta, en cumplimiento de un auto del TSJE, tuvo que rescindir el contrato suscrito entre el Gobierno regional y Petsa.

${ }^{40} \mathrm{Cf}$. "Gallardo compra el $25 \%$ del diario onubense Odiel Información", en El Periódico de Extremadura, 21 de diciembre de 2006; "Polanco vende sus diarios regionales andaluces a Alfonso Gallardo", en El Confidencial, 28 de mayo de 2007 [Consulta: 1 abril 2008]; "Alfonso Gallardo firma un preacuerdo de compra con el Grupo Zeta", en Hoy, 3 de junio de 2008.

${ }^{41} \mathrm{Cf}$. por ejemplo: "Grupo Gallardo aborda el oleoducto en reuniones técnicas con la Junta", 10 de diciembre de 2007, p.15; "Zapatero apoya la refinería Balboa", 7 de marzo de 2008, p.11.
} 

información en Huelva (2006-2008)

prensa llega a un umbral concreto que no suele traspasar: ese umbral es el de la estructura socioeconómica que sostiene a todo un sistema al que conocemos como de economía de mercado".

También apuntaba Reig (1998: 157) que ninguno de los grandes diarios españoles ha conseguido consolidar sus ediciones regionales en Andalucía. Seguramente ésta es la causa por la que Unedisa apostó por la creación de cabeceras locales en unión con grupos empresariales. Huelva Noticias, edición local de El Mundo, fue la primera publicación donde se experimenta esa fórmula de franquicia que, por el momento, parece revelarse como acertada: la estrategia se ha repetido con el nacimiento de la edición local de Almería. Con ésta última son cinco, pues, las cabeceras ligadas a Unidad Editorial -nombre de la empresa editora tras la absorción de Recoletos- en Andalucía: la edición regional andaluza, las locales de Sevilla y Málaga -que dependen directamente del grupo editorial-, y las de Huelva y Almería.

La iniciativa parte de la empresa editora Publicaciones de Huelva, S.A., cuyos mayores accionistas son prácticamente los mismos que avalaron el nacimiento de Huelva Información dos décadas atrás: un "destacado grupo de onubenses" -así se les califica en la presentación del periódico a sus lectores ${ }^{42}$-, presidido por Antonio Ponce, también presidente de la FOE desde hace dieciocho años y vicepresidente de la CEA (Confederación de Empresarios de Andalucía). Al pasar aquel diario a formar parte de sucesivos grupos de comunicación, se hizo necesaria la creación de una nueva cabecera que actuara de correa de transmisión de sus intereses empresariales. El acuerdo establece que las actividades propiamente "periodísticas" -edición, impresión, distribución y promoción, amén de la propia línea editorial- corresponden a Unedisa, mientras que Publicaciones de Huelva asume los gastos de personal e infraestructura.

Huelva Noticias ve la luz el 5 de junio de 2002, y el núcleo de profesionales que lo ha hecho posible en estos años continúa siendo prácticamente el mismo: Rafael Pérez Unquiles en la dirección y José Carlos Aguado como redactor jefe, ambos procedentes de Huelva Información. Junto a ellos, un equipo de más de veinte personas -no todos en plantilla- y tres corresponsales en la provincia. Algunos redactores han ido, en este periodo, siendo promocionados internamente dentro de El Mundo y trabajan ahora en las ediciones de Sevilla y Málaga, lo que acredita un reconocimiento profesional por parte de la empresa. Y realmente la implantación del periódico a nivel local ha ido ascendiendo progresivamente y el número de lectores no deja de crecer $^{43}$.

\footnotetext{
${ }^{42}$ Juan Camacho, "Somos de Huelva", en Huelva Noticias (6 de junio de 2002). Camacho es actual director general de la editora, y este artículo forma parte de un suplemento especial de 96 páginas que el periódico repartió a sus lectores al día siguiente de su nacimiento. En la página 8 aparece la lista completa de accionistas.

${ }^{43}$ Los datos de la OJD (periodo 2006-2007) lo sitúan en un promedio de tirada de 4.693 ejemplares, aunque la difusión es menor que la de Odiel por la estricta política que sigue Unidad Editorial en cuanto al número de tirada: no se conceden suscripciones gratuitas ni siquiera a los colaboradores y el periódico se distribuye casi exclusivamente en venta directa. El número de lectores que acredita el EGM seguramente es más real que en otros diarios.
} 
La línea editorial de Huelva Noticias queda definida en el primer número: junto al sólido aval que presta una cabecera como El Mundo, se marca la diferencia con otros medios resaltando la vinculación local de la empresa editora:

Su principal razón de ser, que no es otra que servir de estímulo y de apoyo al propio progreso de todo lo onubense, definen a la perfección los objetivos: hacer de la unión de estas cabeceras un único periódico en el que la información local y provincial brille con luz propia al lado de la mejor información internacional, nacional y regional [...].No queremos hacer un periodismo pasivo pero tampoco visceral ni beligerante. Defenderemos, con el respeto que todas las ideas merecen, nuestros propios planteamientos y daremos cabida en las páginas de esta cabecera a la opinión plural y acreditada ${ }^{44}$.

Esta defensa de los "propios planteamientos", que naturalmente son los de sus accionistas, ha hecho que en temas tan sensibles como el desmantelamiento del Polo Químico - de nuevo, piedra de toque periodística- el medio se haya posicionado apoyando los intereses de los empresarios. No obstante, hay que reconocerle que, en general, ha cumplido con su responsabilidad informativa para con la sociedad onubense, aunque se tratara de informaciones contrarias a la línea editorial. También en las páginas de opinión han sabido mantener cierta pluralidad. De algún modo, la vinculación con El Mundo ejerce de pantalla protectora para los profesionales, que pueden ofrecer una información más diversa y contrastada; otra cuestión es que las presiones que reciba el medio se diriman a más altos niveles. La misma afinidad ideológica que se establece a nivel nacional explica la postura de Huelva Noticias en los conflictos entre el Ayuntamiento, del PP, y la Diputación, en manos del PSOE; y la batalla que libra el medio contra el proyecto del oleoducto y la Refinería Balboa no puede desvincularse de los ribetes políticos de este asunto.

\section{RADIO}

Las emisoras de radio vinculadas a grandes cadenas son las únicas que existen legalmente en Huelva capital (aparte de los canales públicos, que trataremos más adelante). Estas delegaciones de las cadenas, a su vez integradas en grupos multimedia de comunicación, tienen un margen de maniobra muy reducido: se limitan a elaborar los contenidos de la oferta informativa local, en los periodos previamente asignados en las desconexiones.

Las tres cadenas radiofónicas estatales que operan en Huelva presentan, por lo tanto, un panorama muy similar, incluso en lo que respecta a su historia, funcionamiento y gestión. Las tres emiten a través de dos cadenas ligadas a sus respectivas empresas, una generalista y otra en la modalidad de radio fórmula:

1. La COPE es la primera de las privadas que se instaló en la ciudad, hace ya medio siglo. Las voces y los nombres de los locutores y locutoras que

\footnotetext{
44“"Porque hacía falta”, en Huelva Noticias, 5 de junio de 2002, p.2.
} 

información en Huelva (2006-2008)

durante estas décadas han hablado tras sus micrófonos forman parte de la memoria colectiva de los onubenses, igual que se recuerda a su primer director, el sacerdote José $\mathrm{M}^{\mathrm{a}}$ Roldán, que condujo con mano férrea la emisora hasta bien entrados los 90. La plantilla de COPE en Huelva es de siete personas, algunos de ellos profesionales muy veteranos en la casa. Su director es el periodista Alejandro de Vicente.

2. La SER acaba de celebrar su primer cuarto de siglo de presencia en Huelva, de modo que ha sufrido todos los cambios de titularidad de la cadena a nivel nacional. Radio Huelva es la emisora más escuchada, y existe una delegación local de la Cadena 40 Principales. La plantilla de Cadena Dial Rocío, que emite desde Almonte, también pertenece administrativamente a la SER de Huelva. En total, un equipo de casi veinte personas -la mitad, periodistas-, dirigido por Pedro Neble, hasta hace poco ejecutivo comercial de Odiel Información. Este intercambio de profesionales ha sido habitual entre los medios de Prisa en Huelva.

3. Onda Cero Ha sido la última gran cadena de radio nacional que abrió una delegación en Huelva para la elaboración y emisión de contenidos locales. Antonio Domínguez Risco, su director, coordina también la emisión local de Europa FM y la emisora de Isla Cristina.

A continuación exponemos algunas características que definen al sector radio, desde la perspectiva de los medios de Huelva, a través de las cuales podemos asomarnos al panorama de la radio en España (Reig, 1998 y 2003):

1. Tendencia evidente a la concentración, compensada por la heterogeneidad del sistema: cadenas privadas y públicas, autonómicas o nacionales, además de la presencia de emisoras comunitarias de las que hablamos también más adelante.

2. Crecimiento sostenido y significativo de las cifras globales de audiencia.

3. Los contenidos locales son un complemento necesario que equilibra la continuada apuesta de la radio convencional por la programación en cadena en torno a las grandes estrellas. En estos contenidos predomina, sobre todo, la información, pero también hay lugar para el entretenimiento. Se aprecia, pues, un equilibrio entre lo global y lo local, que es el elemento más significativo de la cultura de la comunicación en la actualidad. La emisión local diaria oscila entre las tres horas de la COPE y las cuatro de la Cadena SER, distribuidas a lo largo del día en cinco o seis informativos, un magazín de mañana, otro de tarde y los programas de deportes. Este horario se reduce los fines de semana.

4. En cuanto a la programación de la radio fórmula no hay demasiadas diferencias entre los contenidos nacionales y locales, si exceptuamos, quizás, la posibilidad de una mayor interacción por parte del público. El tiempo 
para las desconexiones locales es menor que en la radio convencional (alrededor de una hora).

5. El consumo radiofónico se ajusta a los parámetros habituales: el prime time de las emisoras locales se da por la mañana, en días laborables. También gozan de una audiencia significativa los programas de deportes y retransmisiones en directo.

6. Desarrollo lento y tímido de los procesos de innovación tecnológica en las emisoras. La digitalización de la radio ha sufrido un largo retraso a causa del alto coste de los aparatos receptores. La conversión DAB está considerablemente implantada en localidades que concentran una audiencia superior, pero en ciudades como Huelva habrá de esperar aún. Está previsto que a fines de 2011 la señal digital pueda sintonizarse en la mayoría de las capitales de provincia y ciudades de más de 50.000 habitantes, tal como lo recoge el Plan Técnico Nacional de Radio Difusión Sonora Digital. A la espera de este salto, la mayor novedad tecnológica de los últimos años en las emisoras locales ha sido la incorporación de internet, que permite interactuar mucho más con la audiencia.

7. Dura competencia por los ingresos publicitarios: en el caso de la radio conviven las emisoras de estas grandes cadenas con pequeños proyectos radiofónicos, gestionados por instituciones o sociedades tanto privadas como públicas, que atraen a los anunciantes porque mantienen una audiencia significativa.

8. Alta credibilidad del medio radio entre los ciudadanos, que también se aprecia en este ámbito más reducido de lo local. Un detalle significativo es la importancia que concede la clase política local al espacio radiofónico -sobre todo en periodos electorales- como medio idóneo de conectar con la población.

\section{TELEVISIÓN LOCAL}

El actual Estatuto de Autonomía de Andalucía atribuye a la Comunidad autónoma el desarrollo legislativo y la ejecución del régimen de radiodifusión y televisión, competencias que dependen de la Dirección General de Comunicación Social de la Consejería de Presidencia. En cuanto a las radios hay cierta permisividad ante la situación alegal de las emisoras locales, aunque desde el punto de vista institucional sólo se tenga en cuenta la existencia de las cadenas que gozan de licencia administrativa, pero la situación se vuelve más compleja en el caso de la televisión. Y ello no sólo porque el ordenamiento jurídico sea más complicado, sino por el especial momento de cambio que atraviesan estos medios.

En 2008, y tras varias prórrogas, la Junta concedió las licencias destinadas a la iniciativa privada para operar en las demarcaciones locales del mapa andaluz de TDT. Durante los años anteriores, el nerviosismo, las alianzas y los movi- 

información en Huelva (2006-2008)

mientos para posicionarse ante los cambios formaron inevitablemente parte del paisaje de las televisiones locales. Finalmente, de los doce licitadores que fueron admitidos en la demarcación de Huelva ${ }^{45}$, sólo tres, como estaba previsto, lograron la concesión de un canal: Agrupación Radiofónica S.A., Green Publicidad y Medios y Telecuna del Descubrimiento. A pesar de ello, la futura ley audiovisual autoriza la venta y el arrendamiento de las licencias, por lo que el sector de las televisiones locales, a pocos meses ya del célebre apagón, sigue inmerso en su particular batalla digital.

Pese a su consideración legal como servicio público, cada vez se hace más difícil mantener esa ficción en las cadenas audiovisuales, que tienden a someter su libertad al poder económico. Esa es la realidad actual de las televisiones locales en Huelva, que a través de acuerdos con grandes cadenas, o de su sumisión absoluta a los intereses privados, lastran la libertad de información y no digamos el derecho de los ciudadanos a recibir contenidos plurales. Así que, aunque no puedan considerarse en todos los casos auténticas macroestructuras comunicativas (su historia y su titularidad se alejan de lo que venimos atendiendo hasta el momento), tampoco se presentan como pequeñas estructuras independientes del poder. Lo que mostramos a continuación son algunas de las caras de ese "poder" que orientan sobre el significado de los mensajes que se transmiten a través de la televisión local.

En 1984 apareció en el panorama de los medios onubenses la primera televisión local, Odiel TV. Emitía entonces por cable únicamente, y su recepción era muy limitada por parte de la población. Este mismo operador se reconvierte hacia 1993 en una televisión local analógica, de nombre Atlántico TV, que pronto cubre todo el ámbito geográfico provincial por medio de tres antenas. La sociedad titular del medio era, y sigue siendo, Huelva Comunicación Multimedia, SL, desde el principio vinculada con sectores próximos al PSOE. Su consejero delegado es José Luis Jerez Manfredi, quien fuera primer director de Odiel Información.

Durante años Atlántico fue la televisión local por excelencia, y conforme se iba completando el panorama audiovisual de la provincia, los onubenses la identificaban cada vez más con la oposición política en el Ayuntamiento, ya entonces en manos del PP. En 2004 Atlántico y Localia, la agrupación de televisiones locales de Prisa, llegaron a un convenio de emisión conjunta a través de una sociedad intermedia; el pacto suscrito, además de iniciar la reconversión tecnológica de la cadena, concedía a Atlántico la utilización discrecional de la programación de Localia. Pero a fin de adoptar una posición más ventajosa en el tránsito a la televisión digital, desde 2007 Atlántico TV pasó a integrarse en el canal de noticias

\footnotetext{
${ }^{45} \mathrm{La}$ demarcación de Huelva (TLO3H) abarca también los pueblos del cinturón agroindustrial (Beas, Moguer, Palos de la Frontera, San Juan del Puerto y Trigueros); a ella corresponden dos canales de gestión municipal -uno para la capital, ya en funcionamiento, y otro para la agrupación del resto de localidades $-\mathrm{y}$ tres canales privados.
} 
CRN, emitiendo con el nombre de CRN Atlántico46; de este modo, además de optar junto a esta cadena a la concesión de una licencia en la demarcación local, tenía la opción de funcionar como cabecera provincial para las desconexiones si CRN hubiera logrado un canal de TDT autonómico (esta experiencia ya la tuvo con Localia, para la cual la redacción de Atlántico elaboraba los informativos provinciales). Finalmente, CRN no fue seleccionada a nivel regional pero sí resultó ampliamente favorecida en el reparto de concesiones locales: entre ellas, la de Huelva.

Como la audiencia de televisión local no dispone de criterios de medición fiables, lo que pueden ofrecerse son datos acumulativos (número de personas que "pasan" por el canal) facilitados, además, por los propios medios, con lo que estamos obligados a dudar de ellos: hablar de 280.000 espectadores en hora punta puede significar mucho, o nada. La única evidencia es que Atlántico se lleva una buena porción de la tarta publicitaria local. Tal vez eso explica que la empresa Huelva Comunicación Multimedia sea titular también del semanario gratuito La Opinión de Huelva, que se edita desde 1999 y llegó a tener seis cabeceras más en toda la provincia, distribuidas en las poblaciones más importantes. La Opinión cuenta con ingresos publicitarios propios, pero se financia básicamente a través de los beneficios que genera la televisión. Está dirigido personalmente por José Luis Jerez Manfredi y, salvo algunas colaboraciones, se nutre del mismo equipo de profesionales que la televisión. En total trabajan en Huelva Comunicación 23 personas.

La segunda televisión local que comenzó sus emisiones en Huelva fue Teleonuba, y ha sido también la primera en caer víctima de maniobras que obedecen a la actual coyuntura. Teleonuba nació en 1996 por obra y gracia de los mismos accionistas de Huelva Información, quizás como una necesidad de responder a las emisiones de Odiel TV. Desde entonces su historia, y en parte su papel como medio de comunicación, estuvo ligado al diario con el que compartía titularidad, y junto a él pasó por las mismas manos hasta llegar al Grupo Joly. En 2006 fue puesta en venta -la posesión de una televisión local aislada no debía reportarle a Joly mucha rentabilidad- y fue comprada por una productora perteneciente al magnate Kemel Karbachi, a través de la cual se integró en la cadena de televisiones locales de Vocento, Punto TV, cuyo logotipo lució durante dos años. Durante este tiempo se acometió la digitalización del medio. Extrañamente, pese a la inversión realizada, en octubre de 2007 los trabajadores de Teleonuba reclamaban públicamente por medio de un encierro el pago de varios meses de salario y el mantenimiento de la empresa, que consideraban viable. Para entonces Avista TV, la televisión de Vocento llamada a sustituir a Punto TV en Andalucía, ya había

\footnotetext{
${ }^{46}$ Canal Regional de Noticias es propiedad de Green Publicidad y Medios S.A., quien además de optar a 31 concesiones en el concurso TDT local, también presentó su candidatura para gestionar uno de los canales autonómicos privados. Finalmente, las televisiones seleccionadas fueron Avista TV y Corporación Radiofónica, ligadas respectivamente a Vocento y Prisa, en la primera vuelta (marzo de 2007); y Editorial Andaluza de Periódicos independientes (Grupo Joly) y EPI (Prensa Ibérica), en mayo de 2008 .
} 

información en Huelva (2006-2008)

logrado la concesión de uno de los canales autonómicos de TDT, y Teleonuba, finalmente, dejó de emitir sin que nadie pudiera -o quisiera- evitarlo.

La audiencia de Teleonuba la absorbe de momento Antena Huelva, cuya titularidad corresponde a Proartin, una empresa productora de espectáculos con domicilio social en Huelva. Su dueño, el empresario Félix Belzunce, decidió introducirse en el negocio audiovisual y a estos efectos se asoció con Popular $T V$, cuyas emisiones en Huelva eran muy dificultosas a causa de una frecuencia mal sintonizada. La parrilla de programación ofrece así dos franjas claramente divididas, reservándose Antena Huelva el horario de tarde. La empresa solicitó licencia de emisión de TDT en todas las demarcaciones provinciales -en la de la capital, bajo el licitador UTE Televisión Digital Huelva-, no pasó la primera criba, consiguió ser admitida tras la pertinente reclamación en la que acreditaba su solvencia técnica y económica, y finalmente quedó excluida del reparto de licencias. Sin duda, a un sector de la población onubense le interesa la continuidad de esta joven televisión local (emite desde febrero de 2006), que desde la desaparición de Teleonuba, su más directa competidora, ha depuesto el tono políticamente más beligerante que la diferenciaba de aquella. Lo que el futuro depare a Antena Huelva, que ha estrenado nueva gerencia que pretende reconvertir sus contenidos, dependerá de los hipotéticos acuerdos, siempre difíciles por los jugosos ingresos publicitarios en juego, que puedan darse antes de la definitiva puesta en marcha de la TDT en Andalucía.

Muy distinto es el caso de la última televisión local que se ha introducido en el panorama onubense, con fuerza y con un sólido respaldo financiero. Canal Noticias Huelva, $\mathrm{CNH}$, nació en octubre de 2006, aunque su origen está en la emisora de televisión Telecuna del Descubrimiento, que emitía en pueblos limítrofes a la capital desde 1991 y fue adquirida por nuevos accionistas. La inversión millonaria realizada para reconvertir totalmente el medio, y la antigüedad que acreditaba, situó estratégicamente a este operador ante la concesión de licencias digitales de TDT, a la que también optó en la demarcación de la Costa (CNH Costa $)^{47}$.

De todas las televisiones locales de Huelva, $\mathrm{CNH}$ es, sin lugar a dudas, la mejor preparada para el apagón analógico. La calidad de emisión, que es lo primero que aprecian los espectadores, se une a una programación con más de doce horas de contenidos propios, sin acuerdos con otras cadenas y en los que prima la información local. El resultado es una televisión moderna, perfectamente dotada desde el punto de vista tecnológico y con un equipo de profesionales que, entre los dos centros de producción, el de la capital y el de la costa, llega a las 50 personas. La dirección y gestión comercial está en manos de profesionales procedentes de empresas vinculadas a Prisa, y como jefe de los servicios

\footnotetext{
${ }^{47}$ Hay abundante información sobre el medio en el suplemento de 16 páginas que le dedicó Odiel Información al cumplir un año de emisión, el 19 de octubre de 2007. Puede verse en http://www. canalnoticiashuelva.com/index2.html
} 
informativos cuenta con Norberto Javier, hasta septiembre de 2006 presentador y editor del informativo local de Canal Sur ${ }^{48}$.

La titularidad del medio corresponde a la empresa Gesto Comunicación, pero de su accionista mayoritario ya se ha hablado páginas atrás: se trata de Francisco Urbano Gómez, propietario de la constructora Godosa, presidente de la UPECO y copropietario del diario Odiel Información, que en menos de dos años ha conseguido controlar una suculenta parte de los medios locales y vincularse a poderosas empresas de comunicación. ¿Cómo se explica este desembarco tan categórico de Urbano en los medios de comunicación locales? En determinados foros onubenses se ha hablado mucho de la filiación socialista del empresario, dispuesto a dar la batalla en una provincia tan polarizada políticamente como Huelva. Pero sin necesidad de apoyarse en esta explicación, los intereses empresariales de Urbano justifican la anexión de varios medios dentro de una estructura de poder propia. El acceso de Urbano a la cúpula de la UPECO en marzo de 2006 se interpretó como un factor desestabilizador en el escenario de la FOE, ya que la patronal de la Construcción es una de las sectoriales que tiene más peso en la Federación. De hecho, en un movimiento muy significativo, Urbano tanteó después sus posibilidades para hacerse con la presidencia de la misma FOE, controlada por Antonio Ponce. Al ser elegido presidente de la UPECO, Urbano declaró:

Hace falta más consenso y que haya más suelo, barato, para que las viviendas mantengan el precio. La construcción es lo suficientemente importante como para que se nos tenga en cuenta [...]. Nosotros, lejos de ser especuladores, lo que hacemos es crear riqueza y ayudar al desarrollo de Huelva ${ }^{49}$.

Si estas declaraciones fueron hechas en momentos favorables para el sector, sólo preocupado entonces "por la etiqueta de la especulación", cuánto más se hace necesaria preservar la imagen empresarial en una situación de crisis de la industria. Por ello, fundamentalmente, necesita servirse Urbano de los medios de comunicación locales, cuya rentabilidad no se mide sólo en términos económicos. El pequeño conglomerado que ha conseguido gestionar significa la versión local de una realidad cada vez más frecuente en el mundo de la comunicación, la de las empresas o grupos empresariales con ramificaciones en distintos sectores, incluidos los medios de comunicación. Esa estructura, como dice Pascual Serrano (2006: 4), permite que las contabilidades financieras de cada sector no sean independientes y que la inversión en medios no tenga que ser necesariamente rentable, ya que su función prioritaria es atender la nueva imagen del resto de

\footnotetext{
${ }^{48}$ En noviembre de 2005 Esperanza Ruiz, concejal del Ayuntamiento de Gibraleón procedente del PP y esposa de Norberto Javier, otorga con su voto la alcaldía al PSOE tras una moción de censura. Existieron tensiones en la redacción de Canal Sur respecto a la emisión de informaciones sobre el suceso, y Javier se vio envuelto en un conflicto que pudo precipitar su salida del medio.

${ }^{49}$ Inma Gallego, "Los empresarios revalidan hoy la Presidencia de Ponce hasta 2011", en Huelva Información, 1 de febrero de 2007. Véase también Ana Martín, "Upeco renueva su confianza en Francisco Urbano cuatro años más", en Odiel Información, 27 de marzo de 2008.
} 

información en Huelva (2006-2008)

negocios, y de las estructuras ideológicas que permiten mantener el modelo económico que los sustentan. Los medios asumen, pues, su papel en el engranaje socioeconómico, no como "cuarto poder", sino como un subelemento dentro del primero de los poderes: el económico (Reig, 2003: 69).

\section{PEQUEÑAS ESTRUCTURAS INFORMATIVAS EN PRENSA Y RADIO}

Abordamos primeramente el sector de los medios de comunicación de más éxito y crecimiento en los últimos tiempos, el que puede revelarse como más serio competidor de la prensa tradicional: la prensa gratuita. Aunque la dependencia total de la publicidad que ésta mantiene impide que pueda hablarse de auténtico periodismo, la realidad es que tampoco los medios de pago se atreven con la información de verdadera utilidad social, y cada vez más lectores obtienen su información de los medios gratuitos (Reig, 2003: 183).

La provincia de Huelva y su capital, por su estructura social y poblacional, no ofrecen aún mercado para que los buques insignias de la prensa gratuita (20 Minutos, Qué!, Metro, ADN...) se distribuyan en ella, aunque 20 Minutos tiene ya una edición digital de Huelva. Pero el fenómeno, aunque reciente, se ha hecho rápidamente un lugar en la vida cotidiana de la ciudad. De hecho, grupos solventes en la comunicación andaluza han empezado a tomar posiciones, y desde noviembre de 2007 está en la calle el que se denomina a sí mismo, y verdaderamente es, el "primer diario gratuito de Huelva". Se trata de Viva Huelva, un periódico de información general de 24 páginas con una tirada inicial de 20.000 ejemplares. Está editado por Publicaciones del Sur, en línea con la política de expansión de esta empresa editora, que ya tuvo vinculaciones con la prensa onubense ${ }^{50}$ y desde hace años había distribuido otras cabeceras Viva en las más importantes localidades de la provincia. A pesar del previsible déficit, de sus deslices en maquetación y limitaciones de contenido, Viva Huelva cuenta con la enorme ventaja de la escasa competencia.

Y es que tras la prensa gratuita en Huelva sólo hay de momento estructuras informativas pequeñas, pero en cierto modo representativas de tendencias generalizadas en el mundo de la comunicación. Repasaremos brevemente estas iniciativas porque conseguiremos identificar, como nos proponíamos, esos procesos más extendidos en la estructura de la información española.

El maridaje entre publicidad y prensa gratuita convierte estas publicaciones en los hijos perfectos de una ejemplar relación de conveniencia: la del periodismo con las empresas de comunicación e imagen. El paradigma en Huelva lo ofrece Comunicación e Imagen Consorciada, Ceimagen, empresa fundada por un periodista, Fernando Merchán, que tuvo en su momento un papel significativo en

\footnotetext{
${ }^{50} \mathrm{En}$ sus talleres se imprimió, durante su breve andadura, el diario La prensa de Huelva. Véase el amplio listado de semanarios y quincenales Viva en www.publicacionesdelsur.net
} 
la historia de la prensa onubense -fue director fundador de Huelva Información y volvió a pilotar el diario a mediados de los noventa-. Si se tiene en cuenta quiénes eran los accionistas de Huelva Información en esos dos periodos no resultará difícil entender por qué Ceimagen se situó desde el principio en una posición de privilegio en el mercado local de la comunicación, manteniendo excelentes relaciones incluso con la clase política ${ }^{51}$.

El despegue definitivo de Ceimagen se da a principios de esta década, con la puesta en marcha de un portal de seguimiento de medios de comunicación, Seguired (www.seguired.com), que se ha convertido en el tercero a nivel nacional. Seguired es un ejemplo clarísimo del valor -valor en términos económicos- que tiene la información en nuestros días: una información pública que puede ser comercializada porque significa descubrir oportunidades de negocio o vetárselas a la competencia. (Moncada, 2000: 61). Con cifras millonarias de facturación, una plantilla de 80 personas y oficinas por toda España, Grupo Ceimagen sigue siendo una empresa ligada a Huelva que ha diversificado enormemente sus productos y servicios ${ }^{52}$. Así, en 2005, a instancias de los hoteleros de la provincia, saca a la luz Huelva Selección, una publicación gratuita muy cuidada que se distribuye entre una selecta cartera de anunciantes y la clientela de los hoteles. Y a mediados de 2006, pensando en anunciantes con menor capacidad inversora y en la creciente demanda de información deportiva -el Recreativo de Huelva acababa de subir a primera división-, crea Huelva Deportiva.

Este gratuito se distribuye dos veces en semana, y cuando el deporte no ofrece suficiente materia periodística -los sábados, o en periodos no ligueros-, se "desdobla" invirtiendo el sentido de las páginas y acudiendo a la información general que ofrece al reverso Huelva Express. De este modo Ceimagen ha conseguido abrirse a un público distinto, popular si se quiere, y cubrir un sector de mercado más amplio. $Y$ no requiere un esfuerzo ingente, dada la infraestructura con la que ya cuentan. El objetivo, por supuesto, es crecer como empresa: si se puede abarcar ese otro segmento de publicidad, se pueden también captar clientes que en el futuro demanden otros productos a la misma firma porque ya están conectados con ella.

\footnotetext{
${ }^{51}$ Por ejemplo, llevó la campaña electoral que otorgó por primera vez la alcaldía a Pedro Rodríguez, en 1995. El alcalde, empresario y periodista, buen conocedor de los medios de comunicación, había sido accionista de Huelva Información cuando Merchán lo dirigía. Ceimagen ha planificado muchas otras campañas electorales: por ejemplo, la del candidato a la alcaldía de Sevilla por el PP en 2003, Jaime Reynaud. Hay abundante información sobre la empresa en http://www.ceimagen.es/. Interesa particularmente el listado de clientes.

${ }^{52} \mathrm{En}$ la web también pueden verse algunos: desde diseño gráfico a producción de audiovisuales, organización integral de eventos, campañas electorales, etc. Optaron a la concesión de canales de TDT en Huelva, Sevilla y Málaga, y finalmente han obtenido uno en la demarcación de Aracena. Disponen también de una revista digital sobre economía, www.huelvanegocios.com, que se puede descargar gratuitamente.
} 
Una mirada desde lo local: aproximación a la estructura de la información en Huelva (2006-2008)

Hay que decir, sin embargo, que Huelva Deportiva sí vino a cubrir un hueco de información periodística especializada en la ciudad. No existía ningún periódico deportivo sobre el Recreativo que se repartiera en Huelva, ni siquiera había entonces un hábito de lectura de prensa gratuita. La acogida, quizás por la novedad, fue excelente, y llegó a ser la publicación de más tirada de toda la provincia -entre 15.000 y 20.000 ejemplares-. Eso sí, en el interior del Estadio Colombino la competencia era mayor. Este segmento de publicaciones que se reparten entre los asistentes a un partido fue la pionera en Huelva de la prensa gratuita, y las razones son obvias: además del valor en alza de la información deportiva, los anunciantes cuentan con la ventaja de dirigirse a un público escogido y fidelísimo. Huelva Deportiva disputa este espacio, entre otros, con el suplemento Nuevo Colombino, editado por El Mundo Huelva Noticias, y con la revista Recremanía.

Esta última publicación, iniciativa de la pequeña empresa Promotora Onubense de Medios Escritos (Promes), tiene una andadura singular. Promes surge de la inquietud y el entusiasmo por el mundo de la comunicación de sus dos únicos socios, Juan Carlos Jara y Gabriel Tierra, que en sus tiempos de estudiantes fueron los artífices del primer suplemento de información universitaria que existió en Huelva, Facultá, insertado en las páginas de Huelva Información antes de que el diario pasara a manos de Prensa Española; era la época de efervescencia y creación de la Universidad, y este suplemento llegó a tener una tirada aparte que se distribuía gratuitamente en los campus. Pensando en aprovechar la experiencia adquirida, en 2001 estos dos emprendedores pusieron en marcha un tipo de publicación gratuita inexistente en aquel momento, una revista en formato octavo en torno a la información que generaba el Recreativo, que se reparte entre los asistentes a los partidos cuando el equipo juega en casa. La tirada de Recremanía oscila entre 8.000-10.000 ejemplares, y su futuro parece consolidado por la capacidad de movilización social que tiene, cada vez más, el fútbol. Otro tanto ocurre con Campus 21, la revista de Promes especializada en información universitaria, pues la universidad es otro motor social de primer orden en la ciudad. Curiosamente ninguna publicación gratuita había cuajado en la Universidad de Huelva, aunque hubo intentos por parte de Gaceta Universitaria y otras, y este nicho de mercado les animó a poner en marcha el proyecto. En ambos casos tanto los contenidos como el diseño, la maquetación, e incluso la propia gestión de la publicidad dependen exclusivamente de los dos socios de Promes.

Otro tipo de prensa gratuita es la sostenida por una motivación ideológica, de clara filiación política aunque no necesariamente partidaria, cuya expresión más conocida son los semanarios gratuitos de noticias alternativos: periódicos urbanos, irónicos, con una gran carga de opinión y con identidades y enfoques muy heterogéneos, que proliferan en el mercado anglosajón y empiezan a conocerse en España. El medio que nos servirá como ejemplo es La hora de Huelva, un 
gratuito de tendencia progresista. $\mathrm{O}$, como gusta de decir Vicente Toti, uno de sus impulsores, "utópicamente revolucionarios" 53.

La Hora es fruto de los empeños de Toti y otros compañeros con idénticas inquietudes políticas de izquierdas -un colectivo autodenominado "Paz y Naturaleza"- y cierta experiencia en el mundo de la comunicación que están dispuestos a ponerla al servicio de la causa. Aprovechando la infraestructura con la que ya se contaba -la del periódico independiente La voz de Zalamea, que es el antecedente más inmediato de La Hora de Huelva y tiene ya dos décadas de historia-, se configura una mínima estructura redaccional para el medio, cuya pretensión era abarcar la mayor parte de la geografía de la provincia y distribuirse especialmente en la capital. En marzo de 2006 sale a la luz el primer número, cuyo editorial define la línea ideológica de este periódico "de información, formación, ecología y entretenimiento":

Otro mundo es posible (otra manera de informar, formar y entretener también). [...] Alguien tiene que decir basta ya de dictadura con barniz democrático, y encima, tratar de decirlo con la sinceridad, el desenfado, el sarcasmo y la libertad de los que no vivimos, ni pretendemos vivir, del 'pesebre'. La libertad que nos da la no dependencia de ningún grupo mediático ni político.

De La Hora salieron unos doce números, con unos contenidos centrados en temas sociales, políticos y ecológicos, y el casi absoluto predominio del género de opinión. Se le daba cabida también a la literatura y hay que resaltar el lugar preferente otorgado al humor, no sólo presente en las viñetas de Toti u otros dibujantes, sino en la mordacidad e ironía de muchos de los artículos. De esta manera La Hora entronca con esa rica tradición de prensa satírica y popular que se forjó al calor de la exaltación política en el siglo XIX, y hasta su formato de "hoja volandera" lo emparenta con ella. El periódico, sin embargo, dejó pronto de editarse, por agotamiento del proyecto o más bien de las personas que lo sustentaban: el colectivo promotor era responsable no sólo de los contenidos, sino de la maquetación, la distribución -unos 10.000 ejemplares de cada número-, y hasta asumían a fondo perdido los costes de impresión. La actitud refractaria a cualquier forma institucionalizada de organización es uno de los puntos débiles de este tipo de estructuras informativas; lo interesante es que son tan permeables, que en su vulnerabilidad encierran también una capacidad de resurgimiento mucho mayor.

\footnotetext{
${ }^{53}$ Toti es un clásico en la prensa local de Huelva. Es humorista gráfico, trabajó en El Correo de Andalucía durante el periodo de Federico Villagrán, y está vinculado a Huelva Información desde el momento mismo de su fundación: a él se deben el diseño gráfico y la mancheta original del periódico, y en la actualidad continúa publicando allí sus viñetas. Como dibujante y pintor su carrera ha sido ascendente y goza ya de un sólido prestigio. Ha ilustrado numerosos libros, y se ha embarcado en varios intentos editoriales, que es la faceta que nos interesa aquí: fue el responsable de un par de periódicos satíricos independientes y, esporádicamente, de suplementos de arte y ensayo en Huelva Información. Desde hace veinte años dirige La Voz de Zalamea (Zalamea es un pueblo de la cuenca minera).
} 

información en Huelva (2006-2008)

Veamos ahora qué nos ofrece el panorama de la radio no comercial, mucho más versátil que el de la prensa. El bajo coste de producción y la situación de alegalidad consentida que domina el espacio de las ondas ha dado lugar a una maraña de frecuencias, que pueden llegar a alcanzar una cota significativa de audiencia y entran por tanto en la pelea por la tarta publicitaria. Desde los poderes públicos se limitan a recordar que, en caso de conculcación de derechos, no existe sostén jurídico alguno para estas emisoras que operan sin autorización legal.

Ya dijimos que en Huelva no existía ninguna radio municipal, así que las emisoras locales suplen, de alguna forma, esa labor de servicio público. Sí sorprende encontrarse, en cambio, iniciativas radiofónicas vinculadas más o menos abiertamente con empresas que nada tienen que ver con el mundo de la comunicación, tal y como habíamos visto también en los diarios y televisiones locales: seguramente consideran, con razón, que les es más rentable consolidar una emisora que invertir en publicidad en otros medios ${ }^{54}$. Con lo cual se demuestra una vez más que, a este nivel más "modesto" de los medios locales, operan las mismas líneas presentes a nivel mundial y en grandes conglomerados de la comunicación: en este caso, la conexión con empresas de otra naturaleza que a veces son los dueños al cien por cien del medio y culpables de la mayoría de sus prejuicios (Moncada, 2000: 57).

En este prolijo universo de ondas radiofónicas hemos elegido únicamente dos experiencias, que aportan verdaderamente un planteamiento novedoso y constituyen ejemplos muy válidos de las posibilidades de la radio como soporte de una comunicación alternativa. Es evidente que son las menos: lo normal es que en estas radios locales primen los mismos planteamientos mercantilistas que en las grandes cadenas, y que al calor de las ondas surjan incluso locutores que pretenden cierta gloria, en una banal imitación de las figuras mediáticas ${ }^{55}$.

De entre las que se han gestado apostando por esa línea de independencia, Hispanidad Radio constituye toda una referencia en Huelva. Su andadura, ya de más de dos décadas, está ligada a un nombre, el de Juan Infante, cuya historia personal ha escrito parte de la desconocida historia de la radio comunitaria en la provincia. Buscando una protección, ya que no legal, al menos sí institucional, Infante terminó recalando, tras varios experimentos radiofónicos, en la Asociación

\footnotetext{
${ }^{54}$ Baste un nombre como ejemplo: Marismeña Radio (www.marismaradio.com) ha conseguido darse a conocer en la ciudad gracias a una buena cobertura técnica, una programación musical muy pegada a los gustos de su audiencia y un magazín con cierta difusión. Aunque la emisora está constituida como sociedad independiente, su titular -Rafael Puerta- es el mismo que el de una empresa de gestión inmobiliaria, Inmo Presto, y de hecho las instalaciones de la radio comparten domicilio social con las de la inmobiliaria.

${ }^{55} \mathrm{Como}$ el controvertido Paco Morán, locutor deportivo que no duda en aventurarse en otros géneros, y cuyo peculiar y agresivo estilo ha conseguido captar a un sector de público que lo ha seguido en su periplo por diversos medios locales. Actualmente, tras su breve paso por Antena Huelva TV -saldado con una demanda por injurias- continúa con sus retransmisiones deportivas en Antena Huelva Radio, emisora ya desvinculada de su homónima televisiva.
} 
de Vecinos del barrio de La Hispanidad, que actualmente continúa ostentando la titularidad, aunque la emisora ya no depende financieramente de ella. La independencia económica se ha logrado principalmente a través de la publicidad $y$, desde hace poco, a través de convenios esporádicos con el SAE. Con los años Hispanidad Radio ha conseguido constituirse en un espacio radiofónico participativo, de enorme proyección en la populosa barriada que le da nombre y por extensión en toda la capital.

Esta idea de la participación -cada programa nace de un proyecto autogestionado por sus conductores- forma parte de lo que podría llamarse el ideario de la emisora, que da cabida a las aportaciones "tanto de los profesionales como de los ciudadanos de a pie, que tienen cosas que contar, situaciones que plantear e ideas que poner en práctica" ${ }^{156}$. La otra característica del modo de hacer radio de Hispanidad es la preferencia por una radio muy de calle, persiguiendo el desarrollo de los acontecimientos allá donde se producen, con retransmisiones en directo. La realidad es que en eventos deportivos o en las festividades locales, o en cualquier suceso que concentre el interés local, a cualquier hora, hay siempre un locutor de HR, una disponibilidad de la que no gozan las emisoras de las grandes cadenas. Y aunque es imposible contar con datos fiables de audiencia en este tipo de radios no comerciales, la veteranía de la emisora, y hasta la limpieza con la que se sintoniza, le tributa un público fiel y un respetable colchón publicitario.

Con ambos soportes -participación e inmediatez- la radio ha configurado una programación muy abierta, reflejo de su propio objetivo como estructura informativa: "servir de servicio publico, entretenimiento, ocio y cultura, siempre cumpliendo una labor social". Conscientes de la dinámica motivadora que la radio puede ejercer en sectores sociales populares o en colectivos de riesgo, la emisora ha puesto en marcha varios talleres ocupacionales de radiofonía. En ellos participan como monitores algunos de los profesionales de las ondas onubenses que se han curtido en Hispanidad Radio, y de este modo devuelven a la comunidad algo de lo que de ella recibieron.

El último medio al que nos referiremos es la radio de la Universidad de Huelva, Uniradio, (www.uhu.es/uniradio). Como casualmente corresponde, es también el más joven de cuantos aparecen en este artículo, y no sólo por la brevedad de su andadura radiofónica -comenzó a emitir en enero de 2007-, sino por el público al que se dirige y por la originalidad, pluralidad y frescura de sus propuestas. La materialización del proyecto, largamente acariciado, de una radio universitaria, es deudora -como suele suceder- de un empeño personal: el del actual Vicerrector de Tecnología, Innovación y Calidad, el profesor José Ignacio Aguaded.

\footnotetext{
${ }^{56} \mathrm{Cf}$. http://www.hispanidadradio.com. Además de poder oír la programación en tiempo real y de un chat para los oyentes, la página web incluye una breve historia del medio y una declaración de principios en la que HR se alinea junto a las radios comunitarias: "autónoma, independencia de partidos políticos, participación y promoción socio-cultural de los ciudadanos, defensa de los Derechos Humanos, la Paz, el Medio Ambiente y el apoyo a aquellos sectores marginados de la sociedad".
} 

información en Huelva (2006-2008)

Buen conocedor de las posibilidades de los medios, experto en educomunicación y en tecnología educativa, Aguaded ya había participado en un intento anterior de poner en marcha una emisora que sirviera como recurso educativo dentro de la Universidad. Aquella tentativa terminó en fracaso (Aguaded, 2005) y al presentarse de nuevo la oportunidad, el Vicerrector trató de conseguir para la radio una autonomía de funcionamiento y gestión que no la hiciera dependiente de los vaivenes económicos o ideológicos de la universidad. Para asegurar su estabilidad futura se ha madurado un sistema de financiación a través de patrocinadores, lo cual significa un aliciente promocional para empresas e instituciones en una ciudad donde la Universidad goza de un sólido prestigio, y proporciona a la emisora, como contrapartida, un estatus de libertad envidiable.

La estructura redaccional del medio es extremadamente simple: la responsabilidad de la dirección depende de tres personas, pero el grueso de la programación está realizado por los propios oyentes. A principios de curso se abre la convocatoria para acoger aquellas propuestas de realización de programas que, respondiendo a unos requisitos mínimos, llegan desde los distintos sectores de la comunidad universitaria, principalmente de los estudiantes. Por supuesto, este sistema tan participativo tiene sus riesgos. Pero, a la vista de lo ocurrido, ha merecido la pena. En la parrilla de Uniradio conviven espacios musicales de distintos géneros, programas informativos sobre temas sociales, culturales, de entrevistas o de la propia actualidad universitaria. Tal vez sea una radio para minorías, pero está hecha a la medida de sus oyentes, amasada por ellos: en ello radica su diferencia, y puede que su éxito. De hecho, Uniradio ha empezado a captar audiencia fuera de su ámbito de influencia natural, y se oye tanto dentro como fuera de la Universidad. El tiempo dirá, cuando el medio se consolide, si en verdad supone una alternativa a la radio convencional, si tiene auténticamente algo que ofrecer. De momento, en estos primeros años de existencia, la iniciativa ha sido galardonada con numerosos premios que avalan su trayectoria ${ }^{57}$.

En el apartado tecnológico han contado precisamente con el asesoramiento de Hispanidad Radio, a pesar de que ambas ofertas radiofónicas, en parte, se solapen. Seguramente Uniradio ha restado audiencia a Hispanidad Radio en el segmento de público más joven, pero entre ambas emisoras se ha establecido una cooperación más allá de lo cordial, no sólo a nivel técnico -la propia antena de Uniradio está ubicada junta a la de Hispanidad- sino de contenidos. A su modo, entre las radios no comerciales también se producen estrategias de colaboración para sumar fuerzas, sinergias comunicativas que actúan en defensa de la información relevante.

\footnotetext{
${ }^{57}$ Algunos proceden del ámbito universitario (el Premio Esteban S. Barcia de Periodismo Educativo, por ejemplo), y otros han sido concedidos por votación popular (Premio Huelva Joven 2007 del Instituto Andaluz de la Juventud). Además, el programa "Fronteras Abiertas" fue seleccionado por la oficina del Parlamento Europeo en España para representar a España en el Premio Europeo Carlomagno de Juventud en 2008.
} 
Uniradio es un proyecto de radio pionero en Andalucía. Pero aunque aquí sea una práctica novedosa, las radios educativas, particularmente las universitarias, son una experiencia de dilatada trayectoria. El conocimiento directo del tema por parte del profesor Aguaded ha servido para hacer posible el intercambio y el aprovechamiento de muchas de estas experiencias, tanto en España como en Latinoamérica. Con visión de futuro, se ha creado en la Universidad de Huelva una Asociación Universitaria de Radio; de momento, con la intención de poder optar a subvenciones, y a largo plazo, con el fin de desarrollar una Asociación Latinoamericana de Radio Universitaria que tuviera su sede permanente en Huelva.

Una experiencia de comunicación distinta, en fin. $Y$ una programación construida desde abajo, con contenidos donde se equilibran la cultura, los temas de interés social y los espacios de entretenimiento. Lo que demuestra que, cuando no existe la excusa de la "tiranía de la audiencia", es la propia audiencia la que demanda una radio diferente.

\section{CONCLUSIONES}

En cuanto a las grandes empresas informativas, en general, los medios onubenses aparecen vinculados a fuertes grupos de comunicación o establecen alianzas entre ellos, tendentes a fortalecer su permanencia. Se reproduce, en esta óptica de lo local, el proceso de concentración de medios que viene formulándose como una de las tendencias más firmes de la comunicación a nivel mundial. El ejemplo más representativo gira en torno al entramado multimedia que conecta las emisoras de PRISA con el periódico Odiel y las cadenas CRN y CNH, y que tiene, naturalmente, sus conexiones con el poder político. Otra consecuencia inmediata de la concentración es la pérdida de puestos de trabajo.

También es fácilmente perceptible otra tendencia significativa de la información mundial: se tiende a la globalización, pero también a la localización. Todos los medios presentes en este trabajo -también los vinculados a grandes grupos de comunicación- se han plegado a la necesidad de ofrecer una información de cercanía: mayor número de páginas locales en los diarios, espacios informativos en radio y televisión, y una progresiva preferencia por la especialización, sobre todo deportiva. Esta primacía de lo más cercano al ciudadano es la que ha posibilitado la eclosión de las televisiones locales en los últimos años, que en busca de rentabilidad terminan asociándose con cadenas estatales.

El equilibrio de fuerzas entre la concentración y la localización informativa es muy ajustado: si se renuncia a ésta en pos de aquélla, el público buscará otros medios que le proporcionen la información sobre el entorno más inmediato; y en el otro extremo, serán necesarias alianzas que permitan aprovechar las sinergias empresariales y aseguren rentabilidad.

Un ejemplo paradigmático del primer caso lo ofrece la política centralizadora seguida con Odiel Información, que a punto estuvo de sacarlo del mapa de los 

información en Huelva (2006-2008)

periódicos locales. Por otro lado, las alianzas abarcan desde el intercambio de profesionales hasta los acuerdos empresariales de carácter más estable. Sirva como ejemplo lo expuesto sobre las televisiones locales, o la estrategia de Unidad Editorial, de crear cabeceras en unión con grupos de empresarios locales, que parece haber dado resultado en el caso de Huelva Noticias.

La presencia, cada vez más frecuente, de empresas que nada tienen que ver con la información en los medios de comunicación se aprecia también en el ámbito local. La rentabilidad de un medio, más que económicamente, ha de valorarse en términos ideológicos: se invierte en una empresa informativa no para ganar dinero, sino para generar opinión, para ganar en imagen. Huelva ofrece varios ejemplos en este sentido: la FOE en Huelva Noticias, GODOSA en $\mathrm{CNH}$, o el Grupo Gallardo en Odiel. Desde una mentalidad de negocio, y en este terreno más reducido, donde se cuidan menos las formas, un medio de comunicación puede llegar a funcionar como un apéndice sincronizado con un gabinete de comunicación propio.

Las televisiones locales en Huelva presentan un grado de formalización a medio camino entre las macroestructuras informativas (están asociadas a grupos empresariales potentes, o sirven a intereses privados cuyos tentáculos llegan igualmente lejos), y la que corresponde a empresas con menor solvencia económica $\mathrm{y}$, por tanto, con menor calidad en los mensajes que difunden. A pesar de las inversiones realizadas en muchas de las antiguas cadenas locales para asegurar su futuro, el tránsito de la televisión analógica a la digital ha significando un verdadero terremoto cuyos principales afectados son, de nuevo, los profesionales de la información.

En cuanto a las pequeñas o medianas estructuras informativas, de carácter independiente y de titularidad no vinculada a grandes grupos, en su origen, estos medios brotan de tres impulsos o motivaciones: comerciales (una forma de crear beneficios para la empresa), sociales (en el sentido amplio de intereses colectivos a favor de los cuales se pretende intervenir), o lo que podemos llamar "periodísticamente puras": el gusto por informar, por contar lo que pasa y tomarle el pulso a la actualidad.

Hay pequeños medios de comunicación que son consecuencia de iniciativas, empresariales o institucionales, cuyas prioridades van más allá de la información: es el caso de los medios ligados a Ceimagen, puestos al servicio del crecimiento empresarial, o de Uniradio, una emisora de radio educativa. Otras veces, los propios medios son el origen de otras iniciativas que superan también el ámbito de la información: las revistas editadas por Promes configuran ya una empresa con cierta proyección de futuro; la trayectoria de Hispanidad Radio ha dado lugar a una incipiente escuela de radiodifusión; la experiencia de La Voz de Zalamea llevó a sus impulsores a extender su radio de acción a la capital y los pueblos del cinturón. Normalmente estas estructuras son más vulnerables, pues su permanencia suele estar ligada a personas concretas. 
Los medios alternativos no están obligados a generar beneficios, pero sí han de asegurar su rentabilidad económica, indispensable para su permanencia. Esta condición un tanto paradójica ha de ser especialmente tenida en cuenta en medios con motivaciones ideológicas o sociales, si es que desean conservar la independencia o, sencillamente, ser viables.

En cualquier caso, sólo en estas iniciativas encontramos espacio para "los que se informan", que pueden establecer un pacto con "los que informan" para acceder a la información que verdaderamente les interesa. Se detecta a dos niveles. Por arriba, con la adquisición de información especializada por parte de quienes ostentan el poder económico, una información por la que se paga. Por abajo, con la complicidad de los medios que comparten intereses y se abren a las iniciativas de su público. Estaríamos ante algo parecido a lo que se ha venido denominando periodismo cívico: conectar con la comunidad, abrirse a temas e intereses que nunca llegarían a ser noticia, acercar al ciudadano al ámbito de la información.

Pero recordemos, con palabras de Alberto Moncada (2000: 51), que "cuanto más se formalizan los medios de comunicación, menos espacio queda para el ciudadano corriente y para el informador independiente". En Huelva esa "formalización' ha alcanzado ya a las televisiones locales, un sector con bastante complejidad. Sólo en las pequeñas sociedades de prensa o en las radios comunitarias e independientes queda espacio, aún, para la producción de información relevante.

\section{REFERENCIAS BIBLIOGRÁFICAS}

AGUADED, J.I., CORREA, R.I. y GUZMÁN, Ma D. llusiones hertzianas. El Centro de Recursos de Comunicación Multimedia de la facultad de Educación de la Universidad de Huelva, en AA.VV. (2005): // Congreso Iberoamericano de Comunicación Universitaria [en línea]

<http://prensa.ugr.es/prensa/dialogo/biblioteca/cdrom_cicu/cicu2005/com/com-62.pdf>

CHECA GODOY, A. (1991): Historia de la prensa andaluza. Sevilla: Fundación Blas Infante.

EGEA SANTIAGO, C.: "La carrera por la comunicación local (1998-2000): Los grandes' se atreven con 'lo pequeño'”. Ámbitos, (2001), nº 6, pp. 237-260.

MONCADA, A. (2000): Manipulación mediática. Madrid: Libertarias-Prodhufi.

REIG, R. (1998): Medios de comunicación y poder. Prensa, radio, televisión y mundo editorial. Madrid: Paidós.

- (2002): La comunicación en su contexto. Una visión crítica desde el periodismo. Sevilla: Centro Andaluz del libro.

-: Los medios de comunicación al servicio de sí mismos: censura, información, públicos y alternativas. En AA.VV. (2003): Luces en el laberinto audiovisual. Congreso Iberoamericano de Comunicación y educación. Grupo Comunicar/Universidad de Huelva, pp. 177-189.

- (2003): Estructura y mensaje en la sociedad de la Información. Sevilla: Mergablum. 

información en Huelva (2006-2008)

SERRANO, P. (2006): "Una nueva forma de informarse, los medios alternativos". Éxodo, $\mathrm{n}^{\circ}$ 84, pp. 4-9.

TELLECHEA, J.M.: "El conflicto 'info-ambiental': el caso de Huelva Información (agosto de 1983-diciembre de 1992)". Ámbitos, (2004), n 11-12, pp. 319-340.

\section{Breve semblanza biográfica de la autora}

$\mathbf{M}^{\mathrm{a}}$ Ángeles Pastor es Doctora en Filología Hispánica y Licenciada en Periodismo por la Universidad de Sevilla. Ejerce como profesora de enseñanza secundaria y colabora habitualmente en prensa y radio. Fruto de esta doble experiencia son algunos trabajos sobre edu-comunicación. Ha participado en Congresos internacionales y es autora de dos libros.

(Recibido el 01-07-2008, aceptado el 01-03-2009) 